\title{
Consistent apparent Young's modulus of human embryonic stem cells and derived cell types stabilized by substrate stiffness regulation promotes lineage specificity maintenance
}

Anqi Guo ${ }^{1,2}$, Bingjie Wang ${ }^{1,2}$, Cheng Lyu', Wenjing Li ${ }^{1}$, Yaozu Wu ${ }^{3}$, Lu Zhu ${ }^{4}$, Ran Bi ${ }^{1}$, Chenyu Huang ${ }^{5}$, Jiao Jiao $\mathrm{Li}^{6}$ and Yanan Du ${ }^{1 *}$

\begin{abstract}
Background: Apparent Young's modulus (AYM), which reflects the fundamental mechanical property of live cells measured by atomic force microscopy and is determined by substrate stiffness regulated cytoskeletal organization, has been investigated as potential indicators of cell fate in specific cell types. However, applying biophysical cues, such as modulating the substrate stiffness, to regulate AYM and thereby reflect and/or control stem cell lineage specificity for downstream applications, remains a primary challenge during in vitro stem cell expansion. Moreover, substrate stiffness could modulate cell heterogeneity in the single-cell stage and contribute to cell fate regulation, yet the indicative link between AYM and cell fate determination during in vitro dynamic cell expansion (from single-cell stage to multi-cell stage) has not been established.

Results: Here, we show that the AYM of cells changed dynamically during passaging and proliferation on substrates with different stiffness. Moreover, the same change in substrate stiffness caused different patterns of AYM change in epithelial and mesenchymal cell types. Embryonic stem cells and their derived progenitor cells exhibited distinguishing AYM changes in response to different substrate stiffness that had significant effects on their maintenance of pluripotency and/or lineage-specific characteristics. On substrates that were too rigid or too soft, fluctuations in AYM occurred during cell passaging and proliferation that led to a loss in lineage specificity. On a substrate with 'optimal' stiffness (i.e., $3.5 \mathrm{kPa}$ ), the AYM was maintained at a constant level that was consistent with the parental cells during passaging and proliferation and led to preservation of lineage specificity. The effects of substrate stiffness on AYM and downstream cell fate were correlated with intracellular cytoskeletal organization and nuclear/cytoplasmic localization of YAP.

(Continued on next page)
\end{abstract}

\footnotetext{
* Correspondence: duyanan@tsinghua.edu.cn

'Department of Biomedical Engineering, Tsinghua-Peking Center for Life Sciences, MOE Key Laboratory of Bioorganic Phosphorus Chemistry and

Chemical Biology, School of Medicine, Tsinghua University, Beijing 100084,

China

Full list of author information is available at the end of the article
}

SpringerOpen

(c) The Author(s). 2020 Open Access This article is licensed under a Creative Commons Attribution 4.0 International License, which permits use, sharing, adaptation, distribution and reproduction in any medium or format, as long as you give appropriate credit to the original author(s) and the source, provide a link to the Creative Commons licence, and indicate if changes were made. The images or other third party material in this article are included in the article's Creative Commons licence, unless indicated otherwise in a credit line to the material. If material is not included in the article's Creative Commons licence and your intended use is not permitted by statutory regulation or exceeds the permitted use, you will need to obtain permission directly from the copyright holder. To view a copy of this licence, visit http://creativecommons.org/licenses/by/4.0/. The Creative Commons Public Domain Dedication waiver (http://creativecommons.org/publicdomain/zero/1.0/) applies to the data made available in this article, unless otherwise stated in a credit line to the data. 
(Continued from previous page)

Conclusions: In summary, this study suggests that optimal substrate stiffness regulated consistent AYM during passaging and proliferation reflects and contributes to hESCs and their derived progenitor cells lineage specificity maintenance, through the underlying mechanistic pathways of stiffness-induced cytoskeletal organization and the downstream YAP signaling. These findings highlighted the potential of AYM as an indicator to select suitable substrate stiffness for stem cell specificity maintenance during in vitro expansion for regenerative applications.

Keywords: Apparent Young's modulus, Human embryonic stem cells, Substrate stiffness, YAP, Cell fate

\section{Background}

The mechanical properties of cells and associated forces in the cell cytoskeleton are critical elements in mechanochemical signaling pathways, which play a major role in defining fundamental cell functions (Galbraith \& Sheetz, 1998; Vogel \& Sheetz, 2006) including stem cell differentiation (Chaudhuri \& Mooney, 2012). Recently, the elasticity of live cells, which is a fundamental property of cell mechanics and determines the cells' ability to sustain their shape when exposed to mechanical stimuli (Moeendarbary \& Harris, 2014), has been reported to comprise complicated mechanical behavior including viscoelasticity and poroelasticity (Moeendarbary et al., 2013; Hu et al., 2017; Efremov et al., 2017). The elastic properties of a live cell has been classically, universally and sensitively measured by AFM and analyzed by the Hertz model to extract the Young's modulus, which gives a general reflection of the cell's mechanical properties (Rotsch \& Radmacher, 2000). In this work, we defined the apparent elastic modulus of live cells (Dokukin et al., 2013; Collinsworth et al., 2002) directly measured by AFM as the apparent Young's modulus (AYM).

AYM of live cells is mainly determined by the organization of cytoskeletal elements (Fletcher \& Mullins, 2010), and varies among single cells within a population of the same cell type, as well as among different cell types (Butt et al., 2005). There is evidence that the AYM can be potentially used as a label-free indicator of cellular alteration or abnormalities during stem cell development (Yu et al., 2010; Titushkin \& Cho, 2007) or disease pathogenesis (Iyer et al., 2009; Lekka et al., 2012). For instance, the lower rigidity of cancer cells was recently suggested as a marker for cancer diagnosis (Paszek et al., 2005; Lekka \& Laidler, 2009). Other studies have suggested that the AYM may be used as an indicator of cell fate in specific cell types such as ovarian cancer cells (Wenwei et al., 2012) and mesenchymal stem cells (MSCs) (Collinsworth et al., 2002).

To provide optimal cell sources for applications in cell therapy (Li et al., 2010; Li et al., 2014) and establishment of disease models (Yan et al., 2017), it is essential to ensure that the precursor cell types could maintain their desired cell fate during the in vitro expansion process, including characteristics such as self-renewal capacity and maintenance of lineage specificity. As an example, human embryonic stem cells (hESCs) can grow almost indefinitely in vitro and maintain the capacity to differentiate into cells from all three germ layers. hESCs can be induced to differentiate into endoderm cells, which are multipotent and give rise to cells of the gastrointestinal tract (gut, liver, and pancreas), the respiratory system (lung and trachea), and the thyroid (Zorn \& Wells, 2009). Endoderm cells can be further induced to differentiate into bipotent hepatoblasts, which give rise to hepatocytes and cholangiocytes with potential applications in stem cell therapy for acute liver diseases ( $\mathrm{Li}$ et al., 2010) and in vitro cell or tissue models (Yan et al., 2017; Takebe et al., 2013; Camp et al., 2017). At each level of differentiation from hESCs to endoderm cells to hepatoblasts, poorly defined conditions during in vitro cell expansion may lead to the instability of lineage specificity, or 'lineage infidelity' (Ge et al., 2017), which may alter or remove the defining characteristics of these cell types and limit their usefulness for downstream applications. Recent studies have found that in addition to biochemical factors, biomechanical factors in the microenvironment including spatial confinement and extracellular matrix (ECM) stiffness have profound effects on directing cell behavior and fate (Gvaramia et al., 2017). These biomechanical influences can directly impact cell mechanical characteristics including cell stiffness (AYM) and cell shape, leading to changes in the maintenance of pluripotency in stem cells and/or their differentiation into specific lineages (Mathieu \& Loboa, 2012). However, a primary challenge surrounding the application of this knowledge to in vitro cell culture is the ability to apply biophysical cues, such as modulating the substrate stiffness, to regulate AYM and thereby reflect and/or control stem cell lineage specificity for downstream applications.

In our previous work, we investigated the effects of substrate stiffness on cellular heterogeneity during cell expansion from the initial single-cell stage to the resulting multi-cell stage (Wang et al., 2016). We found that the level of variance in hESC heterogeneity in the singlecell stage (prior to in vitro proliferation) directly influenced the percentage of cells that could maintain stemness within hESC colonies in the multi-cell stage (after 
in vitro proliferation). Furthermore, substrate stiffness could modulate cell heterogeneity in the single-cell stage and contribute to regulating cell fate. However, the indicative link between AYM and cell fate determination during in vitro dynamic cell expansion (from single-cell stage to multi-cell stage) has not been established.

It has been previously proved that cells could sense rigidity cues from the substrate and respond through a series of coordinated behaviors, including focal adhesion formation (Pelham \& Wang, 1997; Yang-Kao et al., 2003; Wei et al., 2008; Friedland et al., 2009) and cytoskeletal organization (Tony et al., 2005; Gupta et al., 2015), immediately after passaging onto a substrate with a specific stiffness. YAP, as an important transcriptional factor downstream of the cytoskeletal signaling pathways, is speculated to play an vital role in cell mechanotransduction (Dupont et al., 2011). Cytoskeletal changes involving structural changes in contractile filamentous actin (F-actin) in response to substrate stiffness determine YAP nuclear localization and activity through unidentified molecular effectors (Das et al., 2016). Given the roles of YAP in regulating stem cell self-renewal, differentiation, apoptosis, and cell fate determination (Ian et al., 2010; Kyung-Kwon \& Shin, 2012; Hsiao et al., 2016), it may be an essential element in AYM-related mechanotransduction.

In this study, we established a comprehensive sequential correlation among substrate stiffness, AYM, and cell fate. Specifically, we investigated the previously unanswered questions of: (1) How does AYM change dynamically in different cell types in response to the same change in substrate stiffness? (2) How does the dynamic AYM change pattern in specific cell types correlate with their downstream fate? (3) What are the potential mechanisms involved in cell fate determination when AYM is regulated through substrate stiffness? We hypothesized that during cell expansion, the AYM of the daughter cells needs to be regulated by substrate stiffness to replicate as closely as possible the AYM of the parental cells to ensure lineage specificity, and that these interactions are reliant on YAP-mediated pathways. To systematically investigate the relationships between AYM and substrate stiffness, we employed our previously developed PEGDA hydrogel system to fabricate a series of hydrogels with controlled stiffness, which could provide variations in mechanical stimulation only without accompanying changes in chemical stimulation (Wang et al., 2016). Our findings highlighted the significance of maintaining a consistent AYM in stem cells to preserve lineage specificity for cell-based applications, and provided a new perspective in regulating cell fate through AYM and the mechanotransductive pathways involved.

\section{Results}

AYM changes in epithelial and mesenchymal cell types after passaging onto substrates with different stiffness We tested a series of epithelial (MDCK, HepRG and hESCs) and mesenchymal (3 T3, LX-2 and human adipose-derived MSCs) cell types to systematically investigate dynamic changes in AYM during in vitro culture on substrates with different stiffness (Fig. 1, S2, S3). The substrates used were PEGDA hydrogels with controlled stiffness of $380 \mathrm{~Pa}, 3.5 \mathrm{kPa}$ and $40 \mathrm{kPa}$ produced by regulating UV exposure time (Supplementary Fig. 1a), as well as glass coverslips. The micro-BCA assay showed that there were no significant variations in surface proteins among the selected groups of PEGDA hydrogels, indicating that there were no differences in protein conjugation among these substrates (Supplementary Fig. 1b).

All epithelial cell types showed distinct changes in AYM when passaged onto substrates with different stiffness (Fig. 1a, c). When cultured on rigid substrates, epithelial cells first showed a large increase in AYM at 1 day post-passage, followed by gradually decreasing AYM thereafter. On much softer substrates, epithelial cells first underwent a decrease in AYM at 1 day postpassage, and this decreased AYM was maintained thereafter. On a substrate with 'optimal' stiffness, which was $3.5 \mathrm{kPa}$ for epithelial cells, the AYM of daughter cells was maintained consistently at the same level as the parental cells following passaging and in vitro proliferation. In contrast, the mesenchymal cell types showed different patterns of changes in AYM following passaging onto the same substrates (Fig. 1b, d). Compared to epithelial cells (Supplementary Fig. 2), all mesenchymal cell types had higher parental cell AYM (500-1000 Pa) (Supplementary Fig. 3), which was maintained after passaging onto rigid substrates. On softer substrates, the AYM decreased at 1 day post-passage and was maintained at the same level thereafter.

These results collectively indicated that dynamic changes in AYM occur in both epithelial and mesenchymal cell types following passaging onto substrates with different stiffness. However, AYM responses to changes in substrate stiffness were different between epithelial and mesenchymal cell types and were likely controlled through different mechanisms. Interestingly, while the AYM of epithelial cell types was partly influenced by the increased cell-cell contact at later stages of proliferation, as seen through their AYM changes on rigid substrates at 3-5 days, the AYM of mesenchymal cell types was not dramatically influenced by increased cell-cell contact during proliferation and seemed to only respond to changes in substrate stiffness. Considering these differences and the stiffness range of our hydrogel system, we chose to focus on epithelial cells and particularly hESCs and their derived cell types to investigate the sequential 


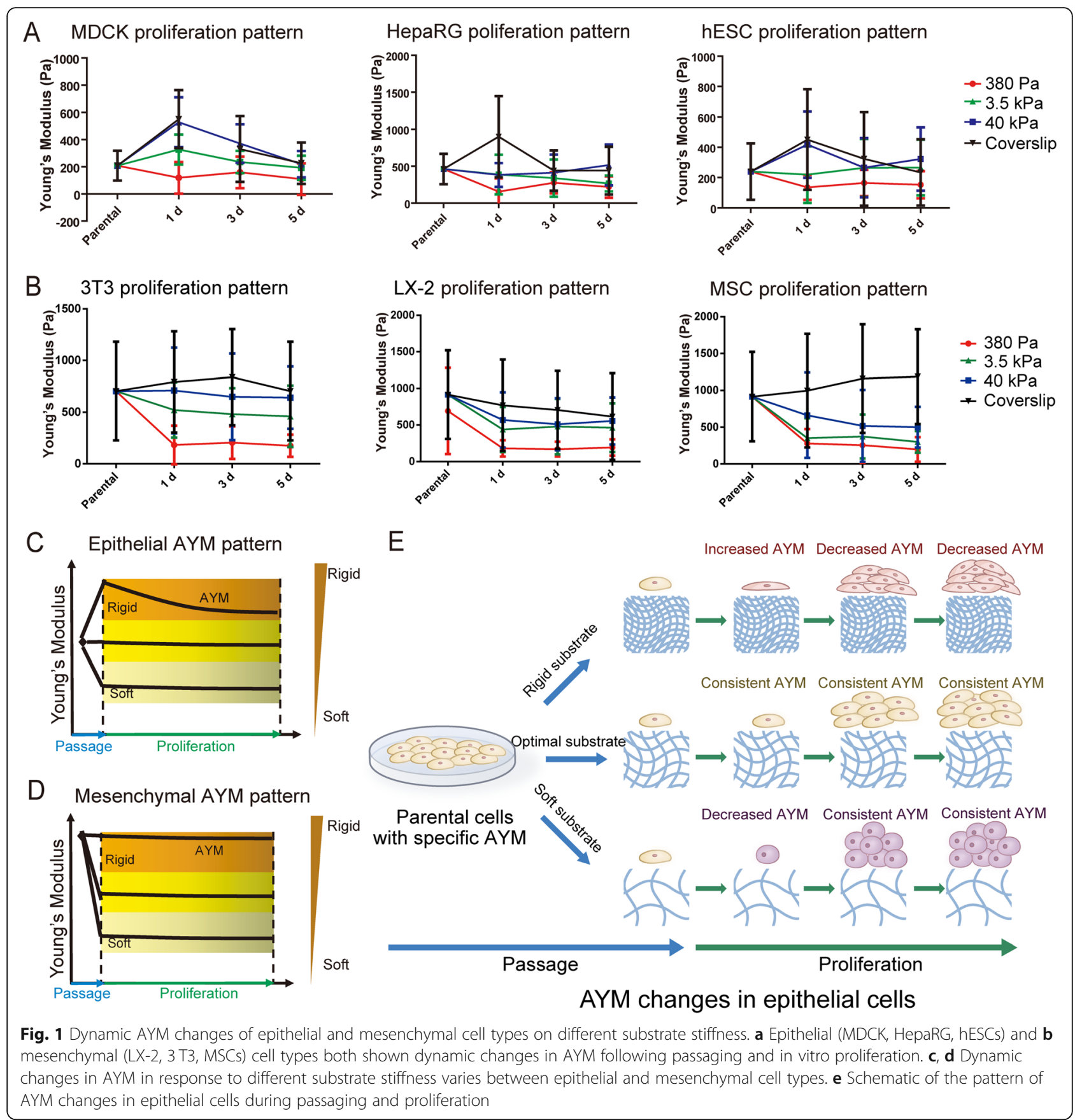

link between substrate stiffness, AYM and cell fate in the rest of the study.

Cytoskeletal organization and focal adhesion formation in epithelial cell types on substrates with different stiffness

The cytoskeletal organization and formation of focal adhesions in response to substrate stiffness were analyzed in hESCs and HepaRG cells as representative human epithelial cell types. The cytoskeletal organization of these cells was evaluated through F-actin distribution.
Cytoskeletal F-actin fibers of parental hESC colonies and HepaRG cells were rounded in shape, with cortical rings and few distinguishable stress fibers (Supplementary Fig. 4). After passaging, epithelial cells cultured on soft substrates $(380 \mathrm{~Pa})$ maintained a rounded shape throughout the culture period, with almost no distinguishable actin stress fibers (Fig. 2a, b). In contrast, cells cultured on rigid substrates (40 kPa hydrogels and coverslips) were wellspread with increasing formation of distinct actin stress fibers during proliferation. Cells cultured on the $3.5 \mathrm{kPa}$ 


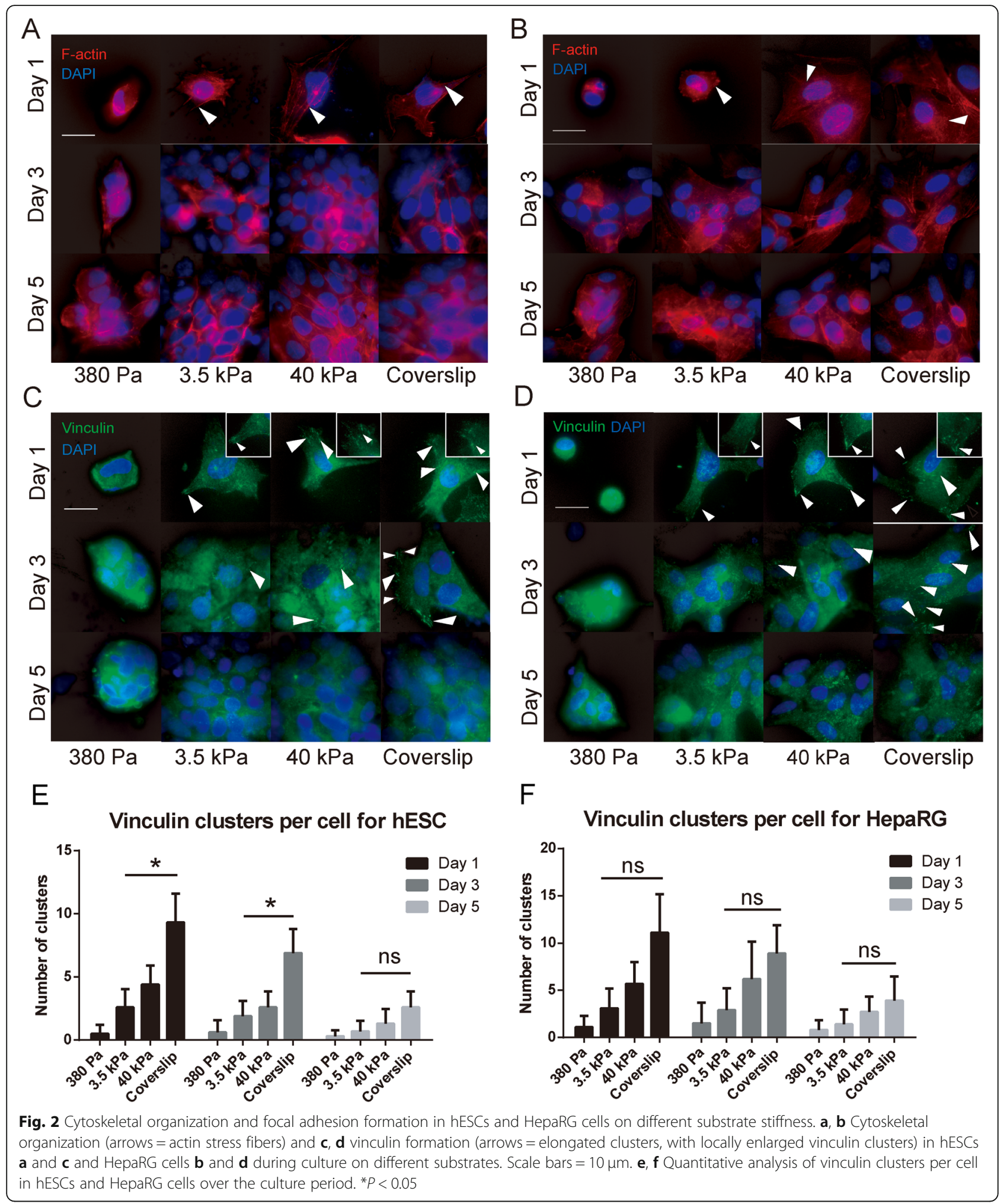

substrate showed some spreading with the formation of a few actin stress fibers during proliferation, and their cytoskeletal structure most closely replicated that of the parental cells.
Vinculin has a key role in focal adhesion formation and its expression was evaluated after the epithelial cells were passaged onto different substrates. The parental hESC colonies and HepaRG cells contained only a few 
vinculin clusters (Supplementary Fig. 4). Low vinculin expression was observed in both cell types after passaging onto the soft $(380 \mathrm{~Pa})$ substrate throughout the culture period (Fig. 2c, d). In contrast, many intense and elongated vinculin clusters formed on the rigid $(40 \mathrm{kPa}$ and coverslip) substrates immediately post-passage, but the number of clusters notably decreased over the 5-day culture period (Fig. 2e, f). Cells grown on the $3.5 \mathrm{kPa}$ substrate showed dispersed vinculin distribution on the cell membrane, and the number of clusters was maintained at almost consistent levels throughout the culture period.

Collectively, these results suggested that while cytoskeletal organization and focal adhesion formation in epithelial cells were influenced by substrate stiffness, daughter epithelial cells growing on substrates with 'optimal' stiffness (i.e. $3.5 \mathrm{kPa}$ ) exhibit similar patterns of cytoskeletal organization and focal adhesion formation as the parental cells, and these patterns are consistently maintained during proliferation. What's more, these patterns are consistent with the AYM change pattern, indicating AYM as an external manifestation of the link between substrate stiffness regulation and cytoskeletal changes in epithelial cells.

\section{Subcellular localization of YAP in epithelial cells on substrates with different stiffness}

YAP is a key mediator of cell mechanotransduction, where its translocation from the cytoplasm to the nucleus in response to increasing extracellular matrix rigidity activates the expression of target genes. The expression level of YAP was analyzed in hESCs and HepaRG cells during culture on substrates with different stiffness. When grown on a soft substrate (380 Pa), YAP in both cell types were mostly located within the cytoplasm over the culture period (Fig. 3a, b, e, f). When grown on rigid substrates (40 kPa, coverslips), YAP was initially localized in the nucleus, but its nucleus/cytoplasm ratio gradually decreased over the culture period. On the $3.5 \mathrm{kPa}$ substrate, the nucleus/cytoplasm ratio of YAP was approximately maintained at a constant level during cell proliferation (Fig. 3a, b, e, f). The trends in nuclear translocation of YAP in response to changes in substrate stiffness were very similar to the changes observed in AYM.

To further investigate the relationship between AYM, cytoskeletal organization and YAP localization, parental hESCs (pre-passage) were grown on the most rigid substrate (coverslip) and treated with $50 \mu \mathrm{M}$ Y27632 (ROCK signaling inhibitor) and $50 \mathrm{mM}$ Blebbistatin (myosin II inhibitor) respectively for $24 \mathrm{~h}$. These small molecule inhibitors disrupt cytoskeletal responses to high substrate stiffness (Supplementary Fig. 5). Correspondingly, treated hESCs maintained a low AYM despite being grown on a highly rigid substrate (Fig. 3c).
Simultaneously, treated hESCs showed YAP translocation from the nucleus into the cytoplasm (Fig. 3d). These findings suggest that changes in AYM reflect dynamic changes in YAP subcellular localization, as a response to variations in substrate stiffness that lead to changes in cytoskeletal organization and focal adhesion formation. AYM can therefore be considered as being correlated with the YAP nucleus/cytoplasm ratio in the response of cells to substrate stiffness changes, as well as to the level of cell-cell contact (Supplementary Fig. 6) which increases during cell proliferation (Fig. 3g).

\section{Maintenance of pluripotency in hESCs on substrates with different stiffness}

The effect of changing substrate stiffness, which is accompanied by changes in AYM, on the maintenance of pluripotency in hESCs was investigated by analyzing their expression of pluripotency markers. After passaging onto hydrogels with different stiffness or coverslips and culturing for 5 days, protein expression of OCT-4 was the most prominent as well as a larger proportion of OCT 4 positive hESCs grown on the $3.5 \mathrm{kPa}$ substrate compared to other groups (Fig. 4a). Fluorescenceactivated cell sorting (FACS) analysis at day 5 also showed that hESCs cultured on the $3.5 \mathrm{kPa}$ substrate was the most homogeneous pluripotent cell population expressing higher level of TRA-1-60 and OCT- 4 compared to other groups (Fig. 4b). Similarly, ALP activity and expression of OCT-4 and NANOG, which represent the stemness maintenance of hESCs, were expressed at the highest level in the $3.5 \mathrm{kPa}$ group at all time points tested (Fig. 4c, d).

When inhibitors were added to block cytoskeletal organization with $10 \mu \mathrm{M}$ Y27632 within the whole 5 days' culture, and block YAP nuclear translocation with $1 \mathrm{uM}$ Verteporfin for $2 \mathrm{~h}$ (removal of Verteporfin in the following 5 days' culture) such that AYM in the hESCs could not change in response to substrate stiffness, the expression level of pluripotency markers OCT-4 and NANOG was no longer significant between groups unlike in the wild-type hESCs (Fig. 4e). Furthermore, by culturing on an optimal substrate stiffness of $3.5 \mathrm{kPa}$ in this study, three consecutive passages of hESCs could all retain better pluripotency characteristics compared with those passaged on coverslips (Fig. 4f, g). These findings suggested that maintaining a consistent AYM by culturing on an optimal substrate stiffness was essential for conserving pluripotency in hESCs during passaging and proliferation.

Maintenance of lineage specificity in definitive endoderm (DE) cells on substrates with different stiffness

We proceeded to investigate whether the same link between substrate stiffness, AYM and maintenance of 

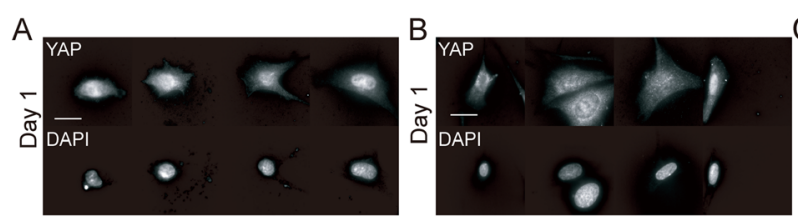

AYM of hESC single-cell stage on coverslip
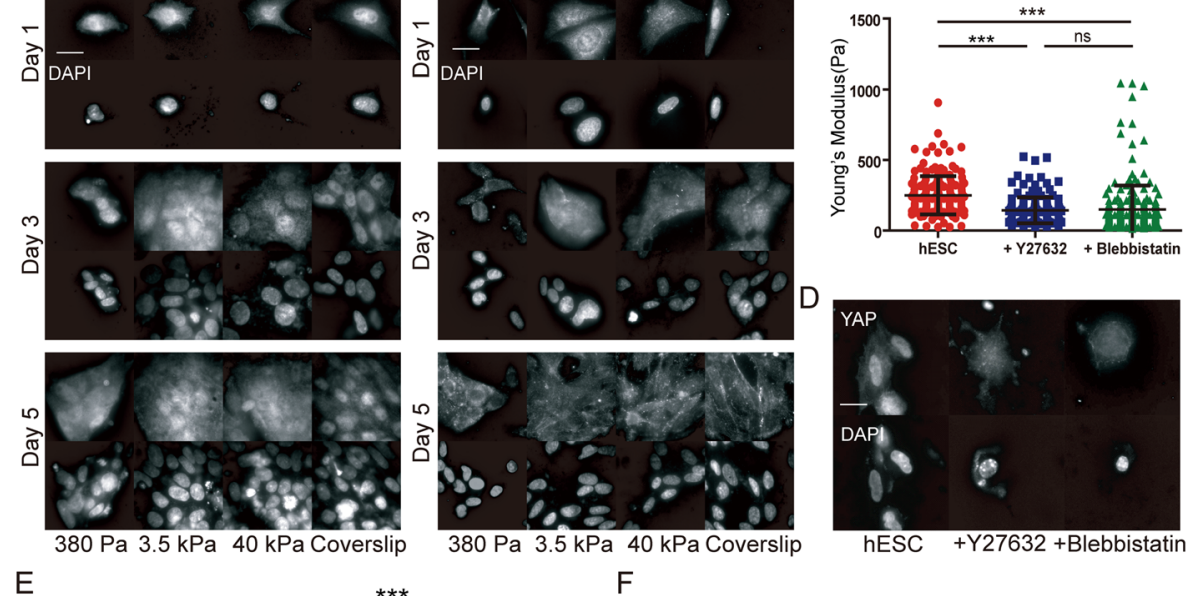

$\mathrm{E}$
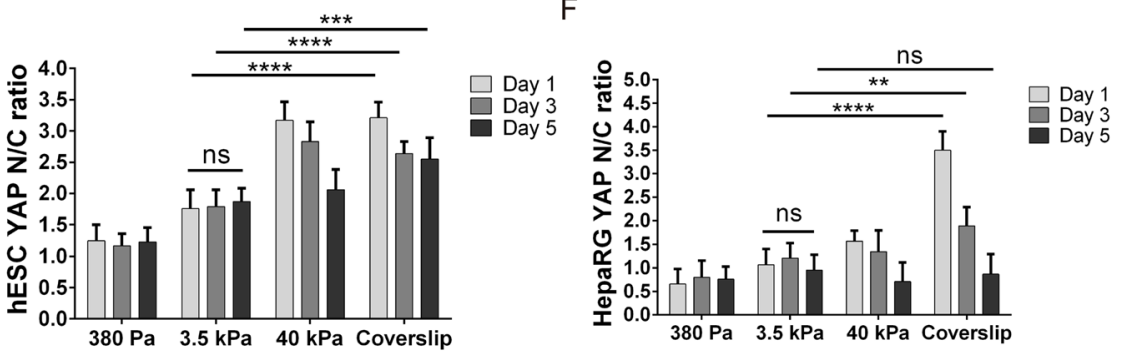

G

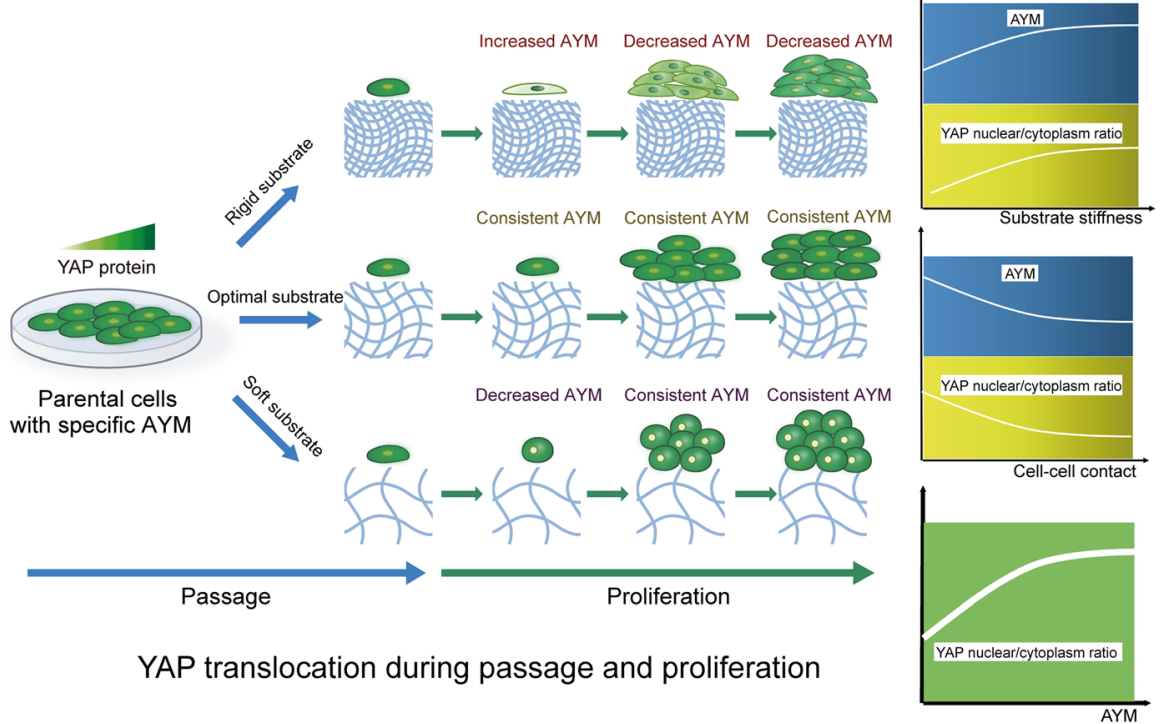

Fig. 3 YAP subcellular localization in hESCs and HepaRG cells on different substrate stiffness. YAP translocation in a hESCs and $\mathbf{b}$ HepaRG cells during culture on different substrates. Scale bars $=10 \mu \mathrm{m}$. $\mathbf{c}$ AYM and $\mathbf{d}$ YAP translocation (nucleus to cytoplasm) of hESCs on coverslip after being treated with Y27632 and blebbistatin. Scale bar $=10 \mu \mathrm{m}$. ${ }^{* *} P<0.001$. Quantitative analysis of YAP nucleus/cytoplasm ratio in $\mathbf{e}$ hESCs and $\mathbf{f}$ HepaRG cells. ${ }^{* *} P<0.01,{ }^{* * *} P<0.001,{ }^{* * * *} P<0.0001$. g Schematics of the relationships between AYM and YAP nucleus/cytoplasm ratio in response to substrate stiffness and cell-cell contact

lineage specificity was present in stem cell types derived from hESCs. Specifically, DE were obtained during the process of hepatic differentiation of hESCs (Fig. 5a), which were used as the parental DE. The AYM of DE with the SOX17-GFP reporter was measured with AFM-total internal reflection fluorescence (TIRF), which provided a more accurate measurement of cell stiffness under its original microenvironment. The AYM of parental DE was measured to be 127.6 Pa (Fig. 5b), which was within the range of epithelial cell stiffness $(<500 \mathrm{~Pa})$ (Supplementary Fig. 2$)$ and allowed the same substrate groups of $380 \mathrm{~Pa}, 3.5 \mathrm{kPa}, 40 \mathrm{kPa}$ hydrogels and coverslips to be applied for testing. 


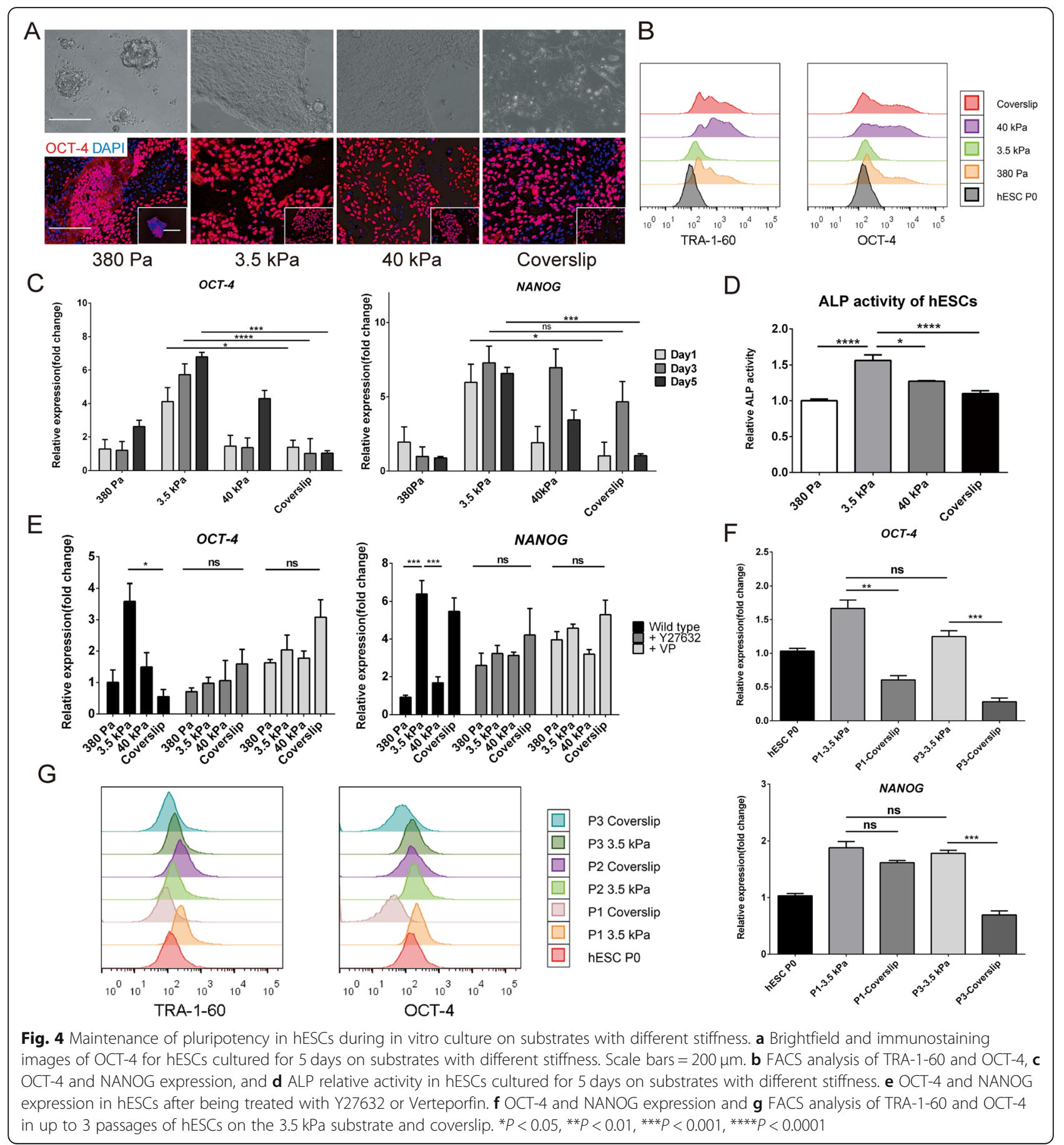

As a lineage-specific marker of DE, SOX-17 was the most highly expressed in cells cultured for 5 days on the $3.5 \mathrm{kPa}$ substrate (Fig. 5c). Compared to parental DE and daughter cells passaged and cultured on other substrates, expression levels of the DE lineage specific markers SOX-17 and FOXA2 on the $3.5 \mathrm{kPa}$ substrate remained the highest (Fig. 5e, g). In contrast, on very soft $(380 \mathrm{~Pa})$ or rigid (coverslip) substrates, a large proportion of cells have completely lost the expression of FOXA2.
When passaged three times, DE showed an elevated growth rate on the $3.5 \mathrm{kPa}$ substrate compared to on coverslips (Fig. 5d), as well as better preservation of SOX17 and FOXA2 gene expression levels between passages (Fig. 5f). DE in the three passages showed an increase in fluorescence intensity through FACS analysis compared to parental cells when grown on $3.5 \mathrm{kPa}$ substrates compared to on the coverslips (Fig. 5h). These findings suggest that substrate stiffness has a similar 


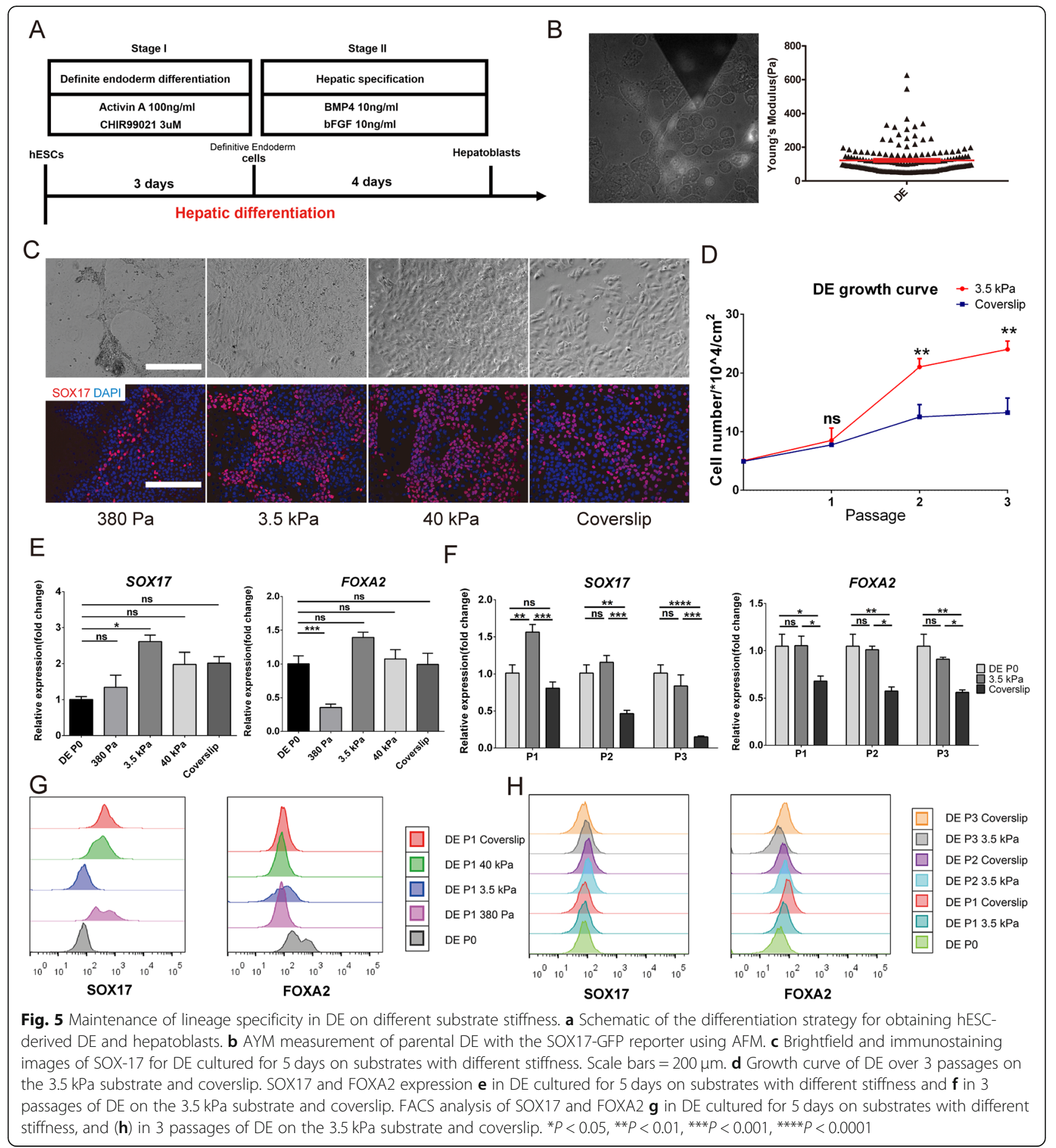

effect on controlling AYM and therefore lineage specificity maintenance of hESC-derived $\mathrm{DE}$ as it does on hESCs.

Maintenance of lineage specificity in hepatoblasts (HBs) on substrates with different stiffness

We further differentiated hESC-derived DE into HBs, which are downstream hepatic progenitors (Fig. 5a). The AYM of HBs with the AFP-mCherry reporter was measured to be $210 \mathrm{~Pa}$ with AFM-TIRF (Fig. 6a), which also fell into the range of epithelial cell types stiffness and allowed the same substrate groups to be applied as in previous experiments. HBs cultured for 5 days on the $3.5 \mathrm{kPa}$ substrate achieved the highest expression of HBspecific markers, as shown through the percentage of AFP-positive cells (Fig. 6b) and expression of PROX1 and AFP (Fig. 6c) compared to other substrate groups. FACS analysis also showed better maintenance of the 

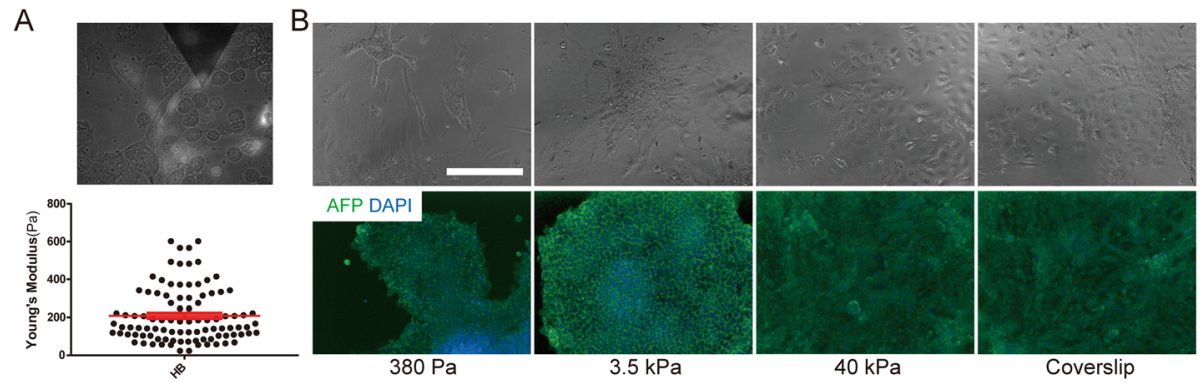

$380 \mathrm{~Pa}$

C
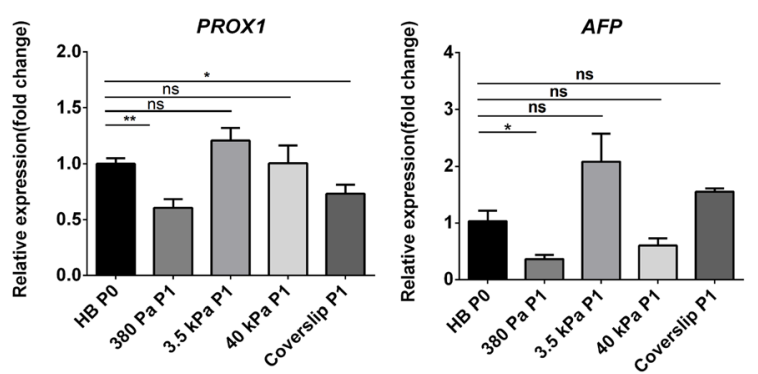

D

E

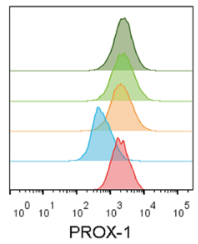

G

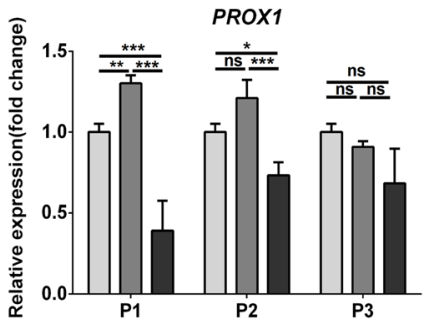

F

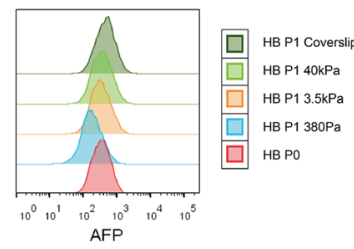

$F$
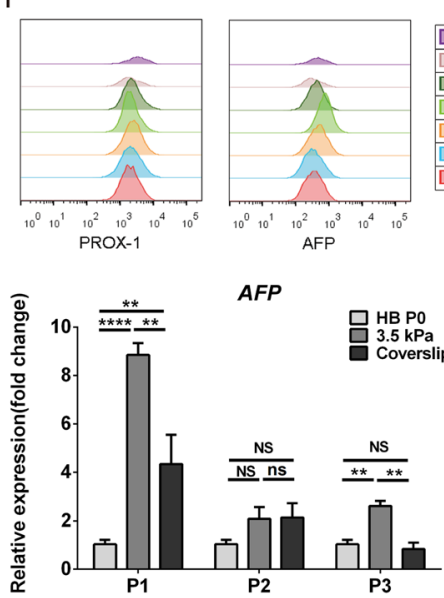

Fig. 6 Maintenance of lineage specificity in HBs during in vitro culture on different substrate stiffness. a AYM measurement of parental HBs with the AFP-mCherry reporter using AFM. b Brightfield and immunostaining images of AFP for HBs cultured for 5 days on substrates with different stiffness. Scale bars $=100 \mu \mathrm{m}$. c PROX 1 and AFP expression in HBs cultured for 5 days on substrates with different stiffness. d Growth curve of HBs over 3 passages on the $3.5 \mathrm{kPa}$ substrate and coverslip. FACS analysis of PROX 1 and AFP e in HBs cultured for 5 days on substrates with different stiffness, and $\mathbf{f}$ in 3 passages of HBs on the $3.5 \mathrm{kPa}$ substrate and coverslip. $\mathbf{g}$ PROX 1 and AFP expression in 3 passages of DE on the $3.5 \mathrm{kPa}$ substrate and coverslip. ${ }^{*} P<0.05,{ }^{* *} P<0.01,{ }^{* *} P<0.001,{ }^{* * * *} P<0.0001$

fluorescence intensity peak in the $3.5 \mathrm{kPa}$ group relative to the parental cells compared to other groups (Fig. 6e).

HBs passaged three times on the $3.5 \mathrm{kPa}$ substrate showed faster proliferation than those on coverslips (Fig. 6d), as well as better expression of PROX1 and AFP at each passage (Fig. 6f, g). Therefore, the lineage specificity maintenance of hESCs-derived HBs could be correlated with AYM stabilization by substrate stiffness regulation, similar to the effects observed in the hESCs and DE.

\section{Discussion}

It is well established that cells sense and respond to substrate stiffness, which influences downstream cell activity and fate (Discher et al., 2005). However, the relation between substrate stiffness and AYM, a primary characteristic defining the mechanical properties of cells, and the influence of changing AYM on cell fate has not been systematically investigated. In this study, we demonstrate that (1) the same change in substrate stiffness causes different patterns of AYM changes in epithelial and mesenchymal cell types, (2) consistent AYM in epithelial cells in response to specific 'optimal' substrate stiffness reflects the maintenance of pluripotency in hESCs and lineage specificity in hESC-derived progenitor cell types, and (3) the effects of AYM changes on cell fate determination is at least partly regulated through 
mechanotransduction by the actin cytoskeleton and subcellular translocation of YAP. Importantly, our findings highlight that for epithelial cell types, better maintenance of lineage specificity can be achieved by controlling substrate stiffness to keep the AYM at a constant level during passaging and cell proliferation, which provides profound implications on optimizing culture conditions for cell therapy, disease modelling, and bio-mechanistic investigations.

Both cell-cell and cell-matrix interactions have critical roles in the mechanical regulation of cell fate. During the single-cell (pre-proliferation) stage where cell-cell interactions are absent, cell-matrix interactions dominate mechano-transductive cell fate regulation. At this stage, cells sense the substrate stiffness through integrin receptors on the cell membrane, and convert this into critical signals that regulate cell lineage specificity (Hansen et al., 1994). These cell-matrix interactions are weakened with cell proliferation, as increased cell density produces more cadherin mediated cell-cell interactions that begin to dominate cell mechanotransduction (Mertz et al., 2013). There is so far little evidence on the synergistic effects of these two types of interactions on regulating cell fate during in vitro expansion. In this study, we found that the AYM could indicate the coordination of cell-matrix and cell-cell interactions during passaging and proliferation, and served as an important 'biomarker' of mechanical regulation that correlated with cell fate determination. Notably, we observed a significant difference in dynamic AYM changes between epithelial and mesenchymal cell types in response to the same changes in substrate stiffness. In epithelial cells, AYM was regulated in the early stage of proliferation by cell-matrix interactions induced by substrate stiffness, and then by cellcell interactions mediated by E-cadherin in the later stages. In mesenchymal cells, AYM regulation was dominated by cell-matrix interactions throughout cell proliferation, which matched the observations of others where fibroblasts could tune their internal stiffness to match their substrate within a defined range (Solon et al., 2007). Unlike epithelial cells, cell-cell interactions had no obvious effects on the fate of mesenchymal cells during passaging and proliferation (Raghu \& Weinberg, 2015; Gheldof \& Berx, 2013). Therefore, our findings illuminated the potential of AYM as label-free indicator to select appropriate substrate stiffness for stemness maintenance of epithelial-like stem cell types during long-term in vitro expansion. Compared to other biochemical indicators with the help of fluorescent antibodies or probes, parental stem cells AYM could be easily measured before passaged onto different substrate stiffness. Then the optimal substrate stiffness, on which daughter cells could maintain similar AYM as parental cells, would be selected for stem cell expansion in vitro. However, in mesenchymal cell types, cell fate determination likely occurs under more complicated mechanisms that only partly involve AYM and requires further investigation.
Substrate stiffness plays a powerful role in regulating cell behavior by modulating focal adhesion formation and cytoskeletal organization, leading to the conversion of mechanical cues into intracellular signals (Alenghat \& Ingber, 2002; Ingber, 2006). YAP is a central player in the mechanosensing pathway and is a downstream transcriptional factor of cytoskeletal F-actin. Our results showed that the nucleus/cytoplasm ratio of YAP was directly influenced by focal adhesion formation and cytoskeletal organization in epithelial cells when grown on substrates with different stiffness. Changes in YAP nucleus/cytoplasm ratio were correlated with changes in AYM, and keeping these parameters at a constant level during proliferation by controlling the substrate stiffness had profound effects on maintaining pluripotency and/ or lineage specificity in epithelial-lineage progenitor cells. When cultured on a rigid substrate such as a coverslip, the high substrate stiffness initially causes YAP translocation from the cytoplasm into the nucleus, which is externally manifested as an immediate increase in AYM. During cell proliferation on the rigid substrate, increased cell-cell contact leads to elevated E-cadherin expression that results in contact inhibition. This is mediated by the Hippo signaling pathway, which causes a cell density-dependent redistribution of YAP from the nucleus to the cytoplasm that inhibits cell proliferation and promotes apoptosis (Nam-Gyun et al., 2011), and manifests as a gradual decrease in AYM during proliferation. In addition, retention of YAP/TAZ complex in the cytoplasm favors the degradation of $\beta$-catenin and inhibits Wnt/ $\beta$-catenin signaling, leading to downstream effects on cell fate including a loss of pluripotency in embryonic stem cells (Azzolin et al., 2014). YAP, as transcription coactivator, must bind to DNA -binding transcription factors such as TEAD to stimulate gene expression (Zhao et al., 2008), since YAP itself has no DNA binding activity. As reported before, YAP was inactivated with increased phosphorylation and cytoplasm translocation during embryonic stem cells differentiation. What's more, YAP and TEAD knockdown lead to a loss of embryonic stem cells pluripotency, while ectopic expression of YAP prevented ES cells differentiation in vitro and maintains stem cell phenotypes even under differentiation conditions, suggesting that YAP and TEAD were required for ES cells pluripotency maintenance. Moreover, YAP bind to promoters of a large number of pluripotent marker genes (including Polycomb group (PcG) proteins, Nanog, Oct4 and Sox2) known to be important for stem cells pluripotency maintenance and stimulated their expression (Lian et al., 2010). In our study, hESCs could maintain AYM and YAP nucleus translocation at a constant level during passage and proliferation on the $3.5 \mathrm{kPa}$ PEGDA-based hydrogel, indicating that the optimal substrate stiffness could 
maintain YAP activation for hESCs self-renewal, while fluctuations of YAP nucleus/cytoplasm ratio on other substrate stiffness indicating the loss of hESCs pluripotency.

Nevertheless, it should be noted that strategies for optimizing the maintenance of cell lineage specificity should not be limited to modulating the AYM. Extracellular matrix components and chemical stimuli in the microenvironment have critical roles in regulating cell fate, and their synergistic effect with mechanical factors should be explored in future studies.

\section{Conclusions}

This study suggests that consistent AYM regulated by optimal substrate stiffness during passaging and proliferation could reflect and effectively contribute to hESCs and their derived progenitor cells lineage specificity maintenance (Fig. 7). Underlying mechanistic pathways involve stiffnessinduced cytoskeletal organization and the downstream YAP nucleus/cytoplasm translocation. Our study provides novel insights for further investigation of mechanistic pathways in mechanobiology, and highlights the significance of AYM as indicators for suitable substrate stiffness selection to maintain stem cells specificity during in vitro large-scale expansion for regenerative applications.

\section{Methods}

\section{Fabrication and functionalization of PEGDA-based} hydrogel substrates

PEGDA hydrogels were prepared using methods modified from our previously published procedures (Wang et al., 2016). Briefly, a precursor solution was made by dissolving
10\% w/v PEGDA, 0.5\% w/v photoinitiator Irgacure D2959 (Insight High Technology Co. LTD, China) and 1\% w/v Nacryloxysuccinimide (NAS) (J\&K, China) in cold phosphate-buffered saline (PBS). The solution was photocrosslinked by UV exposure (OmniCure SERIES 1500, Canada, $20 \mathrm{~mW} \mathrm{~cm}{ }^{-2}$ ) to form hydrogels between a 3-(trimethoxysilyl) propyl methacrylate (TMSPMA)-treated coverslip and an octadecyltrichlorosilane (OTS)-treated glass slide. Different hydrogel stiffness in this study was achieved by adjusting the UV irradiation time ( $380 \mathrm{~Pa}, 3.5$ $\mathrm{kPa}$ and $40 \mathrm{kPa}$ fabricated on TMSPMA-modified coverslips with diameters fitting commercial 24-well plates). Hydrogels were immersed in $75 \%$ ethanol for at least $1 \mathrm{~h}$ for sterilization and removal of un-crosslinked residuals. The hydrogels were functionalized for cell adhesion by coating the surface with different types of proteins at $4{ }^{\circ} \mathrm{C}$ overnight, that is, $0.1 \%$ gelatin (Sigma-Aldrich) dissolved in $\mathrm{diH}_{2} \mathrm{O}$ coated for HepaRG cells, 1:50 diluted vitronectin (Corning) for hESC-derived definitive endoderm (DE) cells, and 1:100 diluted Matrigel (hESC qualified, Corning) for hESCs and hESC-derived hepatoblasts. Surface coated proteins $(0.1 \%$ gelatin coating) on hydrogels of different stiffness were characterized by micro-BCA assay kit (Beotime Biotechnology) according to manufacturer's directions.

\section{Cell culture}

Madin-Darby Canine Kidney (MDCK) cells, HepaRG cells (Life Technologies), and hESCs (H9 line), which represent epithelial cell types, and NIH/3 T3, human hepatic stellate cell line (LX-2) (Xiangya Hospital of Central South University, China), and human adipose-

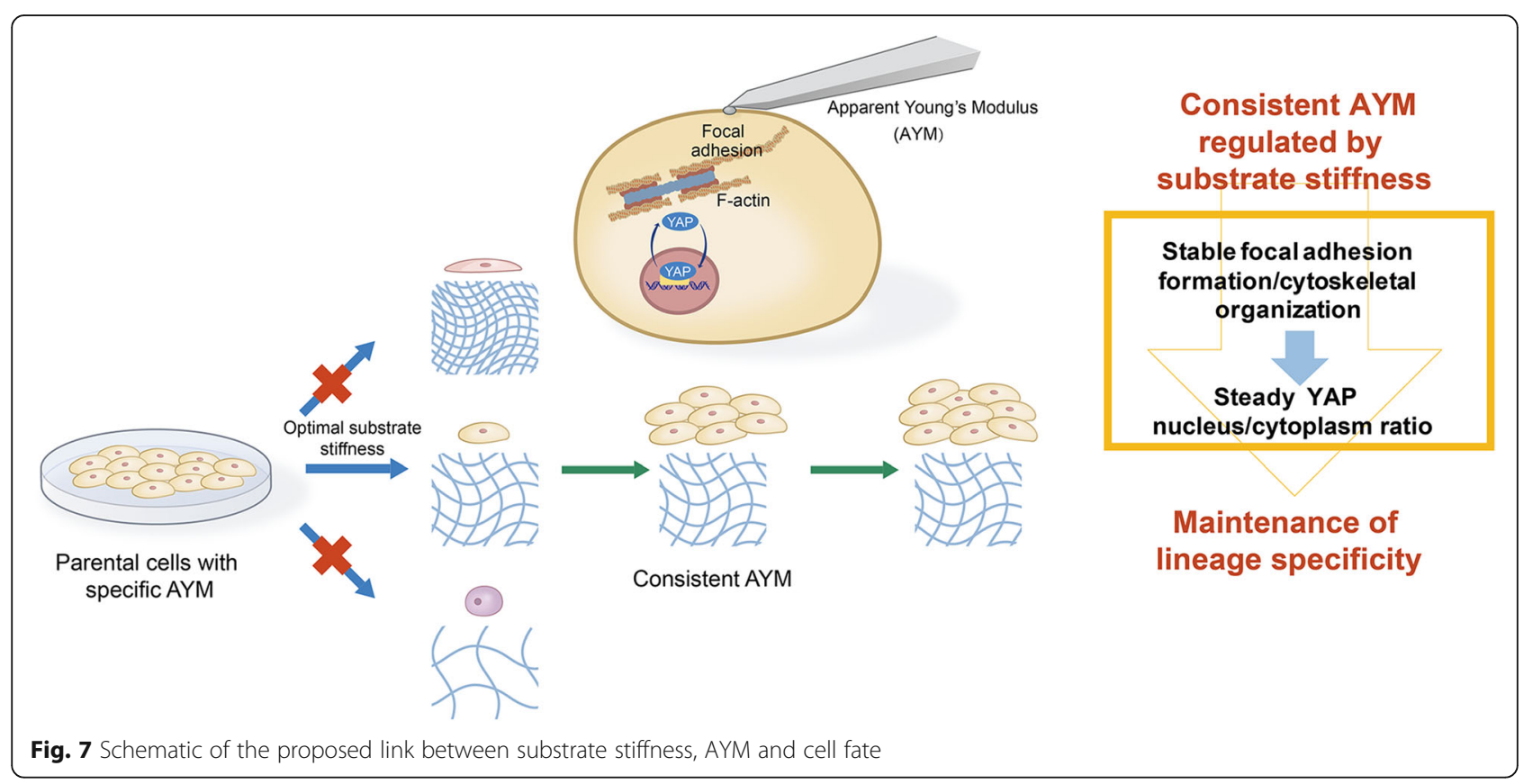


derived mesenchymal stem cells (MSCs), which represent mesenchymal cell types, were used in the subsequent experiments. All cell lines tested negative for mycoplasma. MDCK, 3 T3, LX-2, MSCs and HepaRG were cultured in a humidified $5 \% \mathrm{CO}_{2}$ incubator (Thermo Fisher) at $37^{\circ} \mathrm{C}$. MDCK, $3 \mathrm{~T} 3$ and LX-2 cells were cultured in medium composed of high glucose Dulbecco's modified Eagle medium $(4.5 \mathrm{~g} / \mathrm{L}$ glucose, Wisent, Canada) supplemented with 10\% FBS (Wisent) and $1 \%$ penicillin-streptomycin (Wisent). MSCs were cultured in mesenchymal stem cell growth medium (BioWit Technologies) according to our previous study (Zeng et al., 2015). HepaRG cells were cultured in Williams' E (Gibco) supplemented with 10\% FBS, 1x Glutamax (Invitrogen), $10^{-7} \mathrm{M}$ dexamethasone (DEX) (SigmaAldrich), $5 \mu \mathrm{g} / \mathrm{mL}$ insulin (Aladdin) and $1 \%$ penicillinstreptomycin. H9 hESCs were cultured according to our previously published procedures (Yao et al., 2014). Briefly, cells were maintained under $5 \% \mathrm{CO}_{2}$ at $37^{\circ} \mathrm{C}$ on a feeder layer of $\mathrm{X}$-ray inactivated mouse embryonic fibroblast (MEF) cells and seeded at a density of $2 \times 10^{5}$ / $\mathrm{cm}^{2}$. The medium used for hESCs was KO-DMEM (Gibco) supplemented with 20\% (v/v) KSR (Invitrogen), $1 \mathrm{x}$ GlutaMax, 1x non-essential amino acids (NEAA, Invitrogen) and $8 \mathrm{ng} / \mathrm{mL}$ recombinant human basal fibroblast growth factor (bFGF, Peprotech).

\section{Alkaline phosphatase (ALP) activity assay}

hESCs cultured on $380 \mathrm{~Pa}, 3.5 \mathrm{kPa}, 40 \mathrm{kPa}$ substrates and coverslips for 5 days were subjected to alkaline phosphatase activity analysis with removal of supernatant and addition of an alkaline phosphatase substrate mixture composed of $10 \mathrm{mM}$ diethanolamine (Aladdin), $0.5 \mathrm{mM}$ $\mathrm{MgCl}_{2}$ (Aladdin) and $1 \mathrm{mg} / \mathrm{ml}$ p-Nitrophenyl phosphate (Sigma-Aldrich) in $\mathrm{pH}=10.5 \mathrm{PBS}$. Cells were incubated in the substrate mixture for $2 \mathrm{~h}$ at room temperature. Absorbance was read immediately at $405 \mathrm{~nm}$ on a microplate reader (SpectraMax M5, USA, Molecular Devices). ALP activity was normalized to $1 \times 10^{4}$ cells per sample.

\section{Atomic force microscopy (AFM) for Young's modulus testing and data analysis}

AFM-based mechanical measurements of different cell types were conducted according to our previously published procedures (Wang et al., 2016). Briefly, AFM was performed using the AFM module of Cellhesion200 (JPK instrument, Germany) that is mounted on an inverted optical microscope (Zeiss Observer A1 stand). The AFM probes consisted of a Tipless silicon sensor (ARROWTL1-50, NANOWORLD) with a modified AFM cantilever which had a nominal spring constant of $0.03 \mathrm{~N} / \mathrm{m}$. The cantilever tip was attached with a plain microsphere (6 $\mu \mathrm{m}$ in diameter) to indent the cells for the purpose of simplifying the contact geometry and minimizing the lateral strain of the sample during indentation.

Cells were seeded onto substrates with different stiffness $(380 \mathrm{~Pa}, 3.5 \mathrm{kPa}$ and $40 \mathrm{kPa}$ hydrogels, and coverslip) that were constructed on sterilized $25 \mathrm{~mm}$-diameter coverslips. Prior to cell measurements, the cantilever was first calibrated on the glass coverslip using the thermal vibration method, where the resulting thermal spectrum was fitted with Lorentzian function to determine the spring constant. Individual cells were indented approximately at the center of the cell body (typically sampled over the nucleus) under a piezo-actuated displacement rate of $1 \mu \mathrm{m} / \mathrm{s}$. All AFM measurements were performed in cell medium at $37^{\circ} \mathrm{C}$.

The Young's modulus of each cell was obtained by analyzing the force versus indentation curves using JPKSPM Data Processing software with the classical Hertz model, which was valid for small indentations (approximately up to $5-10 \%$ of cell height or $200-500 \mathrm{~nm}$ ). The minimum number of samples measured was 30 individual cells to ensure accurate measurement for each experimental condition (with multiple indentation locations at the center of each cell body). Poisson's ratio is 0.5 due to the assumption of homogeneity and quasiincompressibility of cells as previously reported (Caille et al., 2002; Ohayon \& Tracqui, 2005).

\section{Differentiation of hESCs into definitive endoderm (DE) and hepatoblasts (HBs)}

hESCs were expanded and also differentiated into DE and HBs for use in subsequent experiments. The hESCs were expanded for 5 days in MEF-conditioned medium (MEF-CM) supplemented with $4 \mathrm{ng} / \mathrm{mL}$ bFGF. For seeding onto fabricated hydrogels, hESC colonies were first disassociated into single cells using Accutase (Gibco) suspended in MEF-CM supplemented with $4 \mathrm{ng} / \mathrm{mL}$ bFGF (Peprotech) and $10 \mu \mathrm{M}$ Y27632 (Medchem Express), followed by seeding onto the hydrogels (precoated with Matrigel at $4{ }^{\circ} \mathrm{C}$ overnight) at a density of $2 \times 10^{4} /$ well in 48 -well plates.

For the differentiation of hESCs into DE, hESCs that were pre-seeded onto Matrigel-coated plates were treated for 3 days with DE differentiation medium, composed of RPMI 1640 basal medium (Wisent) containing 1x B-27 supplement (Gibco), $100 \mathrm{ng} / \mathrm{mL}$ activin A (Peprotech), $3 \mu \mathrm{M}$ CHIR99021 (Medchem Express) and 1x GlutaMax. For further differentiation into $\mathrm{HBs}, \mathrm{DE}$ were treated for another 4 days with $\mathrm{HB}$ differentiation medium, composed of RPMI 1640 basal medium (Wisent) supplemented with $2 \% \mathrm{KSR}$ (Invitrogen), $10 \mathrm{ng} / \mathrm{mL}$ BMP4 (Peprotech), $10 \mathrm{ng} / \mathrm{mL}$ bFGF and $2 \mathrm{mM}$ GlutaMax. Both DE and HB differentiation media were changed daily. 
Table 1 Primary antibodies used for flow cytometry and immunofluorescence staining

\begin{tabular}{lll}
\hline Primary antibody & Manufacturer & Catalog number \\
\hline Human/Mouse Oct-3/4 Antibody & R\&D & AF1759 \\
Purified anti-human TRA-1-60-R & BioLegend & 330,601 \\
Human FOXA2 Antibody & R\&D & AF2400 \\
Human SOX17 Antibody & R\&D & AF1924 \\
Human/Mouse alpha-Fetoprotein/AFP Antibody & R\&D & MAB1368 \\
Anti-PROX1 antibody & Abcam & ab101851 \\
Anti-E Cadherin antibody & Abcam & ab76055 \\
Anti-YAP1 antibody & Abcam & ab52771 \\
Anti-Vinculin antibody & Abcam & ab129002 \\
\hline
\end{tabular}

Re-seeding and expansion of hESC-derived DE and HBs hESC-derived DE and HBs were treated with Accutase and re-seeded onto hydrogel substrates with varying stiffness, which were coated by 1:50 vitronectin for DE and 1:100 Matrigel for HBs. Cells were re-seeded at a density of $5 \times 10^{4} / \mathrm{cm}^{2}$ in medium supplemented with 10uM Y27632. Expansion medium for DE contained DMEM/F12 (Gibco) supplemented with 2\% KSR, $50 \mathrm{ng} /$ $\mathrm{mL}$ bone morphogenetic protein (BMP)-4 (Peprotech), $10 \mathrm{ng} / \mathrm{mL}$ epidermal growth factor (EGF; Peprotech), 10 $\mathrm{ng} / \mathrm{mL}$ vascular endothelial growth factor (VEGF; Peprotech), $50 \mu \mathrm{g} / \mathrm{mL}$ L-ascorbic acid (Sigma-Aldrich), $50 \mu \mathrm{g} /$ $\mathrm{mL}$ Vitamin A (TargetMol), $10 \mathrm{mM}$ nicotinamide (Sigma-Aldrich), 1x GlutaMax and $1 \%$ penicillinstreptomycin. Expansion medium for HBs contained DMEM/F12 supplemented with 10\% FBS, 1x insulin/ transferrin/selenium (ITS, Sigma-Aldrich), $10 \mathrm{mM}$ nicotinamide (Sigma-Aldrich), $10^{-7} \mathrm{M}$ dexamethasone, $1 \mathrm{x}$ GlutaMax, $1 \%$ penicillin/streptomycin, $40 \mathrm{ng} / \mathrm{mL}$ hepatocyte growth factor (HGF; Peprotech) and $20 \mathrm{ng} / \mathrm{mL}$ EGF. Both DE and HB expansion media were changed daily.

\section{Establishment of hESC-SOX17-GFP and hESC-AFP-mCherry reporter lines}

Stable lines of hESC-SOX17-GFP and hESC-AFPmCherry were obtained through lentivirus-mediated infection and screening. Prior to infection, single cells of hESCs were seeded onto Matrigel-coated well plates in
MEF-CM and grown to $40 \%$ confluence, following which concentrated SOX17-GFP or AFP-mCherry lentivirus was added to the medium at a final concentration of $8 \mu \mathrm{g} / \mathrm{mL}$ polybrene supplement. After $8 \mathrm{~h}$ of infection, hESCs were washed twice with Knockout DMEM and kept in normal MEF-CM.

A screening strategy was used to obtain monoclonal stable cell lines. For this, $2 \mu \mathrm{g} / \mathrm{mL}$ blasticidin (BSD, Sigma-Aldrich) was added for 5 days at $48 \mathrm{~h}$ after hESC infection for positive reporter cell screening. Surviving hESCs were then seeded back into the MEF feeder system as single cells until they grew into 10-cell monoclonal colonies. Each monoclonal colony was amplified within a 24-well plate using the MEF feeder system and were differentiated into the $\mathrm{DE}$ or $\mathrm{HB}$ stage. Colonies that showed GFP and mCherry fluorescence were respectively selected as the monoclonal stable hESCSOX17-GFP and hESC-AFP-mCherry cell lines.

\section{Immunostaining, flow cytometry, and real-time quantitative RT-PCR}

Immunostaining was performed according to our previously published procedures (Yao et al., 2014). Briefly, cells were fixed in $4 \%$ paraformaldehyde for $15 \mathrm{~min}$ at room temperature and washed 3 times with PBS. Cells were then permeabilized using $0.5 \%$ Triton, and blocked by PBS containing $1 \%$ bovine serum albumin (BSA). The cells were incubated with primary antibody (diluted

Table 2 Primer sequences used for real-time RT-PCR

\begin{tabular}{|c|c|c|}
\hline Gene & Forward (5'-3') & Reverse $\left(5^{\prime}-3^{\prime}\right)$ \\
\hline OCT4 & CTTGAATCCCGAATGGAAAGGG & GTGTATATCCCAGGGTGATCCTC \\
\hline NANOG & TGGGATTTACAGGCGTGAGCCAC & AAGCAAAGCCTCCCAATCCCAAAC \\
\hline FOXA2 & GGAGCAGCTACTATGCAGAGC & CGTGTTCATGCCGTTCATCC \\
\hline SOX17 & CTCCGGTGTGAATCTCCCC & CACGTCAGGATAGTTGCAGTAAT \\
\hline AFP & TGTACTGCAGAGATAAGTITAGCTGAC & TCCTTGTAAGTGGCTTCTTGAAC \\
\hline PROX1 & CAGATGGAGAAGTACGCAC & CTACTCATGAAGCAGCTCTTG \\
\hline GAPDH & TGCACCACCAACTGCTTAGC & GGCATGGACTGTGGTCATGAG \\
\hline
\end{tabular}


according to the manufacturer's instructions; see Table 1 for details) at $4{ }^{\circ} \mathrm{C}$ overnight, followed by $1 \mathrm{~h}$ incubation with the appropriate Dylight secondary antibody (EarthOx Life Sciences, USA). Cell nuclei were marked with Hoechst 33324 (Beyotime). The stained samples were observed and imaged using a Nikon confocal microscope. For F-actin staining, the fixed and permeabilized samples were exposed to $100 \mathrm{nM}$ rhodamine conjugated phalloidin (Cytoskeleton, USA) for $30 \mathrm{~min}$ and Hoechst 33342 (Sigma-Aldrich) for $10 \mathrm{~min}$ at room temperature, followed by imaging using a Nikon Eclipse $\mathrm{Ti}-\mathrm{S}$ microscope. ImageJ plot profile tool and Imaris surface tool were used for image quantification.

For flow cytometry, cells were dissociated into single cells using $0.25 \%$ Trypsin/EDTA and incubated in blocking buffer containing $1 \%$ goat serum for $30 \mathrm{~min}$, followed by incubation in primary and secondary antibodies according to the manufacturers' instructions. The cells were analyzed using a BD LSRFortessa SORP flow cytometer (BD Biosciences). Three independent samples were examined for each condition, and approximately $10^{5}-10^{6}$ cells were counted for each sample.

For gene expression analysis, total RNA was extracted using TRIzol reagent (Invitrogen), followed by reverse transcription using first-strand cDNA synthesis kit (Takara), according to the manufacturers' instructions. Expression levels of all genes were quantified using SYBR Green II (Vazyme) on a Bio-Rad CFX96 Real-Time PCR platform, and normalized to the housekeeping gene glyceraldehyde 3-phophate dehydrogenase (GAPDH; see Table 2 for primer sequences) using the comparative $\mathrm{Ct}\left(2^{-\Delta \Delta \mathrm{CT}}\right)$ method.

\section{Statistical analysis}

Statistical analysis was performed in GraphPad Prism using two-way analysis of variance in conjugation with one-way ANOVA and pairwise multiple comparison tests. All data were presented as mean \pm standard deviation (SD). Differences were considered statistically significant for $P<0.05$. Data were obtained from at least three independent samples unless otherwise stated.

\section{Supplementary information}

Supplementary information accompanies this paper at https://doi.org/10. 1186/s13619-020-00054-4.

\footnotetext{
Additional file 1: Supplementary Figure 1. (A) UV exposure time and the corresponding hydrogel Young's modulus measured by AFM. (B) Surface coated protein characterization by micro-BCA assay for hydrogels of different stiffness. NC represented the coverslip without protein functionalization. ${ }^{* * *} P<0.0001$. Supplementary Figure 2. Numerical values of AYM for epithelial cell types on substrates with different stiffness. AYM of (A, B) MDCK, (C, D) HepaRG, (E, F) hESCs when cultured on substrates with different stiffness at 1, 3 and 5 days, as well as the AYM of parental and daughter cells at day 1 . Supplementary Figure 3. Numerical values of AYM for mesenchymal cell types on substrates with different stiffness. AYM of (A, B) 3T3, (C, D) LX-2, (E, F) MSCs when
}

cultured on substrates with different stiffness at 1, 3 and 5 days, as well as the AYM of parental and daughter cells at day 1 . Supplementary Figure 4. Focal adhesion, cytoskeletal organization and YAP localization in (A) hESC and (B) HepaRG parental cells. Supplementary Figure 5. hESCs treated with Y27632 and Blebbistatin showed dissipation of Factin, suggesting disabled cytoskeletal responses to increase in substrate stiffness. Supplementary Figure 6. Significant increase in E-cadherin expression of hESCs during 5 days of in vitro proliferation on a rigid substrate (coverslip).

\section{Abbreviations}

AYM: Apparent Young's modulus; YAP: Yes-associated protein; hESCs: Human embryonic stem cells; DE: Definitive endoderm; HBs: Hepatoblasts;

AFM: Atomic force microscopy

\section{Acknowledgements}

We thank L. Zhang's lab (Tsinghua University, Beijing, China) for providing the MDCK cells, Q. Shen's lab (Tsinghua University) for providing the $\mathrm{NIH} / 3$ T3 cells, and Beijing Tsinghua Changgung Hospital (Beijing, China) for providing human adipose tissue for isolating the MSCs. We thank colleagues from the School of Medicine, Tsinghua University: Prof. Yan Shi and Dr. Xia Tie for assisting with AYM characterization using the AFM-TIRF system, as well as Prof. Jie Na and Prof. Kehkooi Kee for assisting with hESCs culture.

\section{Authors' contributions}

A.G., B.W. and Y.D. conceived and designed the experiments; A.G. performed most of the cell passaging, aPCR analysis, flow cytometry and

immunofluorescence staining experiments; B.W. assisted in apparent Young's modulus testing by AFM; C.L. assisted in flow cytometry analysis; W.L. assisted in schematics design and production; Y.W. and L.Z. assisted in fabrication and functionalization of PEGDA-based hydrogel substrates; A.G., B.W. and Y.D. wrote the manuscript, which R.B., C.H. and J.L. helped to revise, Y.D. is the principal investigator of the supporting grants. All authors have given approval to the final manuscript.

\section{Funding}

This work was financially supported by the Beijing Municipal Science\& Technology Commission (Z181100001818005), the National Natural Science Foundation of China (31671036) and Beijing Natural Science Foundation (JQ18022) and the Australian National Health and Medical Research Council (APP1120249)

\section{Availability of data and materials}

The datasets analysed during the current study are available from the corresponding author on reasonable request.

\section{Competing interests}

The authors declare that they have no competing interests.

\section{Author details}

'Department of Biomedical Engineering, Tsinghua-Peking Center for Life Sciences, MOE Key Laboratory of Bioorganic Phosphorus Chemistry and Chemical Biology, School of Medicine, Tsinghua University, Beijing 100084, China. ${ }^{2}$ School of Life Sciences, Tsinghua University, Beijing 100084, China. ${ }^{3}$ Department of Biomedical Engineering, McKelvey School of Engineering, Washington University in St. Louis, St. Louis 63130, USA. ${ }^{4}$ Institute of Systems Engineering, Academy of Military Sciences, Beijing 100071, China. ${ }^{5}$ Department of Dermatology, Beijing Tsinghua Changgung Hospital, School of Clinical Medicine, Tsinghua University, Beijing 102218, China. ${ }^{6}$ Kolling Institute, University of Sydney, Sydney, NSW 2006, Australia.

Received: 21 April 2020 Accepted: 15 July 2020

Published online: 03 September 2020

\section{References}

Alenghat FJ, Ingber DE. Mechanotransduction: All Signals Point to Cytoskeleton, Matrix, and Integrins. Sci Signal. 2002; 2002: pe6.

Azzolin L, Panciera T, Soligo S, Enzo E, Bicciato S, Dupont S, et al. YAP/TAZ incorporation in the $\beta$-catenin destruction complex orchestrates the Wnt response. Cell. 2014;158:157-70. 
Butt H-J, Cappella B, Kappl M. Force measurements with the atomic force microscope: technique, interpretation and applications. Surf Sci Rep. 2005;59:1-152.

Caille N, Thoumine O, Tardy Y, Meister J-J. Contribution of the nucleus to the mechanical properties of endothelial cells. J Biomech. 2002;35:177-87.

Camp JG, Sekine K, Gerber T, Loeffler-Wirth H, Binder H, Gac M, et al. Multilineage communication regulates human liver bud development from pluripotency. Nature. 2017;546:533-8.

Chaudhuri O, Mooney DJ. Stem-cell differentiation: anchoring cell-fate cues. Nat Mate. 2012;11:568.

Collinsworth AM, Zhang S, Kraus WE, Truskey GA. Apparent elastic modulus and hysteresis of skeletal muscle cells throughout differentiation. Am J Physiol Cell Physiol. 2002;283:C1219-C27.

Das A, Fischer RS, Pan D, Waterman CM. YAP nuclear localization in the absence of cell-cell contact is mediated by a filamentous actin-dependent, myosin Iand Phospho-YAP-independent pathway during extracellular matrix Mechanosensing. J Biol Chem. 2016;291:6096-110

Discher DE, Janmey P, Wang Y-L. Tissue Cells Feel and Respond to the Stiffness of Their Substrate. Science. 2005;310:1139.

Dokukin ME, Guz NV, Sokolov I. Quantitative study of the elastic modulus of loosely attached cells in AFM indentation experiments. Biophys J. 2013;104:2123-31.

Dupont S, Morsut L, Aragona M, Enzo E, Giulitti S, Cordenonsi M, et al. Role of YAP/TAZ in mechanotransduction. Nature. 2011;474:179-83.

Efremov YM, Wang W-H, Hardy SD, Geahlen RL, Raman A. Measuring nanoscale viscoelastic parameters of cells directly from AFM force-displacement curves. Sci Rep. 2017;7:1541.

Fletcher DA, Mullins RD. Cell mechanics and the cytoskeleton. Nature. 2010;463: 485-92.

Friedland JC, Lee MH, David B. Mechanically activated integrin switch controls alpha5beta1 function. Science. 2009;323:642-4.

Galbraith CG, Sheetz MP. Forces on adhesive contacts affect cell function. Curr Opin Cell Biol. 1998;10:566-71.

Ge Y, Gomez NC, Adam RC, Nikolova M, Yang H, Verma A, et al. Stem Cell Lineage Infidelity Drives Wound Repair and Cancer. Cell. 2017;169:636-50 e14.

Gheldof A, Berx G. Cadherins and epithelial-to-mesenchymal transition. Prog Mol Biol Transl Sci. 2013;116:317.

Gupta M, Sarangi BR, Deschamps J, Nematbakhsh Y, Callan-Jones A, Margadant F, et al. Adaptive rheology and ordering of cell cytoskeleton govern matrix rigidity sensing. Nat Commun. 2015;6:7525.

Gvaramia D, Müller E, Müller K, Atallah P, Tsurkan M, Freudenberg U, et al. Combined influence of biophysical and biochemical cues on maintenance and proliferation of hematopoietic stem cells. Biomaterials. 2017;138:108-17.

Hansen LK, Mooney DJ, Vacanti JP, Ingber DE. Integrin binding and cell spreading on extracellular matrix act at different points in the cell cycle to promote hepatocyte growth. Mol Biol Cell. 1994;5:967-75.

Hsiao C, Lampe M, Nillasithanukroh S, Han W, Lian X, Palecek SP. Human pluripotent stem cell culture density modulates YAP signaling. J Biotechnol. 2016;11:662-75

Hu J, Jafari S, Han Y, Grodzinsky AJ, Cai S, Guo M. Size- and speed-dependent mechanical behavior in living mammalian cytoplasm. Proc Natl Acad Sci. 2017;114:9529.

lan $\mathrm{L}$, Joungmok $\mathrm{K}$, Hideki $\mathrm{O}$, Jiagang $Z$, Bin $Z$, Jindan $Y$, et al. The role of YAP transcription coactivator in regulating stem cell self-renewal and differentiation. Genes Dev. 2010;24:1106-18.

Ingber DE. Cellular mechanotransduction: putting all the pieces together again. FASEB J. 2006:20:811-27.

lyer S, Gaikwad RM, Subba-Rao V, Woodworth CD, Sokolov I. Atomic force microscopy detects differences in the surface brush of normal and cancerous cells. Nat Nanotechnol. 2009;4:389-93.

Kyung-Kwon L, Shin Y. Identification of mechanism that couples multisite phosphorylation of yes-associated protein (YAP) with transcriptional coactivation and regulation of apoptosis. J Biol Chem. 2012;287:9568-78.

Lekka M, Gil D, Pogoda K, Dulińska-Litewka J, Jach R, Gostek J, et al. Cancer cell detection in tissue sections using AFM. Arch Biochem Biophys. 2012;518:151-6.

Lekka M, Laidler P. Applicability of AFM in cancer detection. Nat Nanotechnol. 2009;4:72.

Li F, Liu P, Liu C, Xiang D, Deng L, Li W, et al. Hepatoblast-Like Progenitor Cells Derived From Embryonic Stem Cells Can Repopulate Livers of Mice. Gastroenterology. 2010;139:2158-69 e8.

Li Y, Liu W, Liu F, Zeng Y, Zuo S, Feng S, et al. Primed 3D injectable microniches enabling low-dosage cell therapy for critical limb ischemia. Proc Natl Acad Sci. 2014;111:13511.
Lian I, Kim J, Okazawa H, Zhao J, Zhao B, Yu J, et al. The role of YAP transcription coactivator in regulating stem cell self-renewal and differentiation. Genes Dev. 2010;24:1106-18.

Mathieu PS, Loboa EG. Cytoskeletal and focal adhesion influences on mesenchymal stem cell shape, mechanical properties, and differentiation down osteogenic, adipogenic, and chondrogenic pathways. Tissue Eng Part B-Re. 2012;18:436-44.

Mertz AF, Che Y, Banerjee S, Goldstein JM, Rosowski KA, Revilla SF, et al. Cadherin-based intercellular adhesions organize epithelial cell-matrix traction forces. Proc Natl Acad Sci U S A. 2013;110:842-7.

Moeendarbary E, Harris AR. Cell mechanics: principles, practices, and prospects. WIREs Syst Biol Med. 2014;6:371-88.

Moeendarbary E, Valon L, Fritzsche M, Harris AR, Moulding DA, Thrasher AJ, et al. The cytoplasm of living cells behaves as a poroelastic material. Nat Mater. 2013;12:253-61.

Nam-Gyun K, Eunjin K, Xiao C, Gumbiner BM. E-cadherin mediates contact inhibition of proliferation through hippo signaling-pathway components. Proc Natl Acad Sci U S A. 2011;108:11930-5.

Ohayon J, Tracqui P. Computation of adherent cell elasticity for critical cell-bead geometry in magnetic twisting experiments. Ann Biomed Eng. 2005:33:131-41.

Paszek MJ, Zahir N, Johnson KR, Lakins JN, Rozenberg Gl, Gefen A, et al. Tensional homeostasis and the malignant phenotype. Cancer Cell. 2005;8:241-54.

Pelham RJ, Wang YL. Cell locomotion and focal adhesions are regulated by substrate flexibility. Proc Natl Acad Sci U S A. 1997;94:13661-5.

Raghu K, Weinberg RA. The basics of epithelial-mesenchymal transition. J Clin Invest. 2015;119:1420-8.

Rotsch C, Radmacher M. Drug-induced changes of cytoskeletal structure and mechanics in fibroblasts: an atomic force microscopy study. Biophys J. 2000;78:520-35.

Solon J, Levental I, Sengupta K, Georges PC, Janmey PA. Fibroblast adaptation and stiffness matching to soft elastic substrates. Biophys J. 2007;93:4453-61.

Takebe T, Sekine K, Enomura M, Koike H, Kimura M, Ogaeri T, et al. Vascularized and functional human liver from an iPSC-derived organ bud transplant. Nature. 2013:499:481-4

Titushkin I, Cho M. Modulation of cellular mechanics during osteogenic differentiation of human mesenchymal stem cells. Biophys J. 2007;93:3693-702.

Tony Y, Georges PC, Flanagan LA, Beatrice M, Miguelina O, Makoto F, et al. Effects of substrate stiffness on cell morphology, cytoskeletal structure, and adhesion. Cell Motil Cytoskel. 2005;60:24.

Vogel V, Sheetz M. Local force and geometry sensing regulate cell functions. Nat Rev Mol Cell Biol. 2006;7:265-75.

Wang B, Qin P, Zhao H, Xia T, Wang J, Liu L, et al. Substrate stiffness orchestrates epithelial cellular heterogeneity with controlled proliferative pattern via Ecadherin/ $\beta$-catenin mechanotransduction. Acta Biomater. 2016:41:169-80.

Wei WC, Lin HH, Shen MR, Tang MJ. Mechanosensing machinery for cells under low substratum rigidity. Am J Physiol-Cell Ph. 2008;295:C1579.

Wenwei X, Roman M, Byungkyu K, Lijuan W, John MD, Todd S. Cell stiffness is a biomarker of the metastatic potential of ovarian cancer cells. PLoS One. 2012 7:e46609.

Yan F, Wang $Y$, Zhang W, Chang M, He Z, Xu J, et al. Human embryonic stem cell-derived hepatoblasts are an optimal lineage stage for hepatitis $C$ virus infection. Hepatology. 2017;66:717-35.

Yang-Kao W, Yao-Hsien W, Chau-Zen W, Junne-Ming S, Wen-Tai C, Shu-Han L, et al. Rigidity of collagen fibrils controls collagen gel-induced downregulation of focal adhesion complex proteins mediated by alpha2beta1 integrin. J Biol Chem. 2003;278:21886-92.

Yao R, Wang J, Li X, Jung Jung D, Qi H, Kee KK, et al. Hepatic differentiation of human embryonic stem cells as microscaled multilayered colonies leading to enhanced homogeneity and maturation. Small. 2014;10:4311-23.

Yu H, Tay CY, Leong WS, Tan SCW, Liao K, Tan LP. Mechanical behavior of human mesenchymal stem cells during adipogenic and osteogenic differentiation. Biochem Biophys Res Commun. 2010;393:150-5.

Zeng Y, Chen C, Liu W, Fu Q, Han Z, Li Y, et al. Injectable microcryogels reinforced alginate encapsulation of mesenchymal stromal cells for leakproof delivery and alleviation of canine disc degeneration. Biomaterials. 2015; 59:53-65.

Zhao B, Ye X, Yu J, Li L, Li W, Li S, et al. TEAD mediates YAP-dependent gene induction and growth control. Genes Dev. 2008;22:1962-71.

Zorn AM, Wells JM. Vertebrate endoderm development and organ formation. Annu Rev Cell Dev Biol. 2009;25:221-51. 